\title{
Eficacia de las normas de bioseguridad frente a la pandemia del COVID-19
}

\author{
Effectiveness of biosecurity standards in the face of the COVID-19 \\ pandemic
}

Eficacia de las normas de bioseguridad

\author{
Carmen Lisseth Anchundia Mero ${ }^{1}$ \\ Elvira Geoconda Villacreses Veliz Ms. Ge ${ }^{2}$ \\ Virginia Esmeraldas Pincay Pin $^{3}$ \\ ${ }^{1}$ Universidad Estatal del Sur de Manabí, Jipijapa, Ecuador \\ ${ }^{2}$ Universidad Estatal del Sur de Manabí, Jipijapa, Ecuador \\ ${ }^{3}$ Universidad Estatal del Sur de Manabí, Jipijapa, Ecuador
}

Contacto: elvira.villacreses@unesum.edu.ec

Recibido: 15-07-2021 Aprobado: 10-08-2021

\begin{abstract}
Resumen
El trabajo de investigación identifica la bioseguridad como técnicas aplicadas con el fin de evitar la exposición no intensional a patógenos y toxinas, el problema científico de esta investigación está basado en las medidas de bioseguridad y la importancia del correcto uso del equipo de protección personal, ya que estos insumos son indispensables para disminuir el riesgo de contagio por COVID-19 y proteger la salud tanto del paciente como del profesional. El objetivo general del proyecto de investigación es determinar el cumplimiento de las normas de bioseguridad en la prestación de servicios del personal sanitario para evitar contagios por COVID-19 en el distrito de salud 13D03, el estudio realizado fue descriptivo de corte transversal, aplicando el método analítico-sintético para la correcta realización y abordaje de la investigación, además de técnicas de recolección de datos como encuestas y entrevistas. La gran mayoría de los profesionales sanitarios del Distrito de Salud 13D03 aplican las medidas de bioseguridad en su ambiente de trabajo y labores diarias, mientras que una pequeña parte solo las aplica a veces, debido a la falta de disponibilidad de insumos y equipos de protección personal en los centros de salud, se evidenció al inicio de la pandemia, además de que existe limitación por parte del distrito en la utilización de los EPP completos de manera conjunta y continua, la aplicación de las normas de bioseguridad son sin duda una parte esencial para la lucha contra el coronavirus evitando el contagio masivo entre la población y profesionales de salud.
\end{abstract}

Palabras clave: contagio, equipos de protección personal, insumos, patógenos, toxinas

\section{Summary}

The research work identifies biosafety as applied techniques in order to avoid non-intensive exposure to pathogens and toxins, the scientific problem of this research is based on biosafety measures and the importance of the correct use of personal protective equipment, since that these supplies are essential to reduce the risk of contagion by COVID-19 and protect the health of both the patient and the professional. The general objective of the research project is to determine compliance with the biosafety standards in the provision of services of health personnel to avoid contagion by COVID-19 in the health district 13D03, the study carried out was descriptive of cross-section, applying the method analytical-synthetic for the correct conduct and approach of the investigation, in addition to data collection techniques such as surveys and interviews. The vast majority of health professionals in Health District 13D03 apply biosafety measures in their work environment and daily tasks, while a small part only apply them sometimes, due to the lack of availability of supplies and personal protective equipment. in health centers, it was evident at the beginning of the pandemic, in addition to the fact that there is a limitation on the part of the district in the use of complete PPE jointly and continuously, the application of biosafety standards are undoubtedly an essential part of the fight against the coronavirus avoiding massive contagion among the population and health professionals. 
HIGÍA DE LA SALUD

Keywords: contagion, personal protective equipment, supplies, pathogens, toxins

\section{Introducción}

A diario el personal sanitario se encuentra propenso a contraer todo tipo de infecciones y enfermedades, por el contacto directo que tienen con los pacientes en la atención de los mismos, por este motivo es indispensable el cumplimiento de las normas de bioseguridad y uso adecuado del equipo de protección personal, para disminuir el riesgo de contagio.

La Organización Mundial de la Salud define a la Bioseguridad como aquellos principios, técnicas y practicas aplicadas con el fin de evitar la exposición no intencional a patógenos y toxinas, mientras que la bioprotección, como aquellas medidas de la institución y del personal destinadas a reducir el riesgo de pérdida, robo, uso incorrecto, desviaciones o liberación intencional de patógenos y toxinas (1); y en la actualidad es de suma importancia la aplicación de las mismas por parte del personal de salud para evitar o disminuir contagios por la pandemia del coronavirus por parte del personal sanitario.

En el sector salud las instituciones, requieren del establecimiento, planificación y cumplimiento de un programa de bioseguridad, como parte esencial de su organización técnica y administrativa de funcionamiento; el cual debe involucrar objetivos y normas definidos que consigan un ambiente de trabajo ordenado, seguro, confiable y que conduzcan simultáneamente a mejorar la calidad de atención, reducir sobrecostos hospitalarios y alcanzar los niveles óptimos de funcionalidad en estas áreas, haciendo referencia al principio de bioseguridad como es la universalidad (2).

Estudios en el área de salud y seguridad en el trabajo a nivel mundial, demuestran que la exposición laboral a infecciones agudas o crónicas, causadas por diversos agentes, especialmente virus, hongos y bacterias, en áreas como urgencias, quirófanos, entre otros son factores de riesgo para la salud del trabajador y de la comunidad (3).

Según datos de la Organización Internacional de Trabajo el número de accidentes y enfermedades relacionados con el ambiente laboral, que anualmente cobra más de 2 millones de vida, parece estar aumentando debido a la rápida industrialización de algunos países (3), por lo que el riesgo de contraer una enfermedad o que ocurra un accidente laboral es el peligro más frecuente a los que se exponen el personal de salud y por lo tanto, es necesario que se cumplan con los protocolos de bioseguridad e igualmente las instituciones estén dotadas de implementos de protección personal.

Actualmente el personal de salud tanto de instituciones de salud pública como privadas en todos los niveles de
ISSN 2773-7705

Periodo. Julio - Diciembre 2021

Vol. 5, Nro. 2, Publicado 2021-12-31

atención están luchando contra una pandemia mundial denominada COVID-19, mediante la cual se han modificado y reforzado protocolos de bioseguridad de todo el mundo.

La pandemia por COVID-19 ha desafiado todos los componentes del sistema de salud: gobernanza, financiamiento, servicios de salud, insumo y equipamiento, sistema de información y recursos humanos (4). También ha puesto en evidencia problemas estructurales e históricos de los sistemas de salud. Así, aun los sistemas de salud mejor preparados para una pandemia sufrieron colapso parcial o completo (5).

En Ecuador el Ministerio de Salud Pública elaboró un Manual llamado "Bioseguridad para los establecimientos de salud" publicado en el 2011, con actualización en el 2016, el mismo que tiene como objetivo estandarizar las medidas de bioseguridad para disminuir el riesgo de los trabajadores de salud y también de las enfermedades relacionadas a la atención sanitaria (6).

En el presente año 2020 en conjunto con la Secretaria Nacional de Garantía de Calidad de los Servicios de Salud y la Dirección Nacional de Calidad de Servicios de Salud, el

MSP elaboró los Lineamientos de prevención y control para casos SARSCOV2/COVID-19.

Esto busca fortalecer las medidas de prevención y control de infección del COVID-19 incluido el uso adecuado y racional de los equipos de protección personal en los diferentes ámbitos de acción hospitalario, ambulatorio, laboratorio, pre-hospitalario, puntos de entrada, cuidados domiciliarios y espacios públicos, que son un factor clave para contener la transmisión a nivel local y comunitario (7).

Las normas generales y procedimientos de este documento son de aplicación obligatoria para todos los trabajadores y profesionales de la salud del Ministerio de Salud Pública y para la Red Pública Integral y Red Privada Complementaria de Salud, en tanto guarden conformidad con las disposiciones legales, reglamentarias y resoluciones vigentes al momento de ejecutarse los respectivos lineamientos (7).

Anteriormente se ha mencionado al coronavirus, pero ¿Qué es? ¿De dónde proviene? ¿Por qué es tan importante la aplicación de las normas de bioseguridad en el personal sanitario y la población?

Los coronavirus son una extensa familia de virus que pueden causar enfermedades tanto en animales como humanos (7), en los humanos pueden ir desde un simple resfriado hasta un síndrome agudo respiratorio severo. En diciembre del 2019 se encontraron varios casos de neumonía inexplicables relacionado con el mercado mayorista de marisco del Sur de China en Wuhan (8), el cual es muy visitado y conocido por la comercialización de especies exóticas, en donde días posteriores científicos manifestaron encontrar en dichos pacientes 
HIGÍA DE LA SALUD

una cepa de coronavirus el cual se le denominó SARSCOV2.

Esta denominación responde a las siglas en ingles Severe Acute Respiratory Syndrome SARS, COV por tratarse de un coronavirus, ya conocido desde la década de los años sesenta e identificada su existencia desde la prehistoria en animales, y 2 porque es diferente al SARSCOV identificado ya años antes (9).

Con toda esta información científicos de Wuhan manifestaron, que el nuevo coronavirus fue una transmisión zoonótica a humanos, y la información genética encontrada proviene de los murciélagos, los cuales son comercializados vivos y consumidos como platillos exóticos por los habitantes de esa región, por lo que los primeros contagios ocurrieron en el mercado de Wuhan.

Este virus se transmite entre las personas a través de gotas respiratorias y vías de contacto como fómites en el entorno inmediato alrededor de la persona infectada (7) por lo que existen tres formas de transmisión: por gota, contacto y aire; y es que a pesar de que el COVID-19 tiene una baja tasa de mortalidad, su taza de virulencia es alta por lo que su propagación fue rápida y en menos de 2 meses pasó de solo encontrarse en la ciudad de Wuhan a toda China.

El cuadro clínico que presenta es muy parecido a otros cuadros de problemas respiratorios de origen vírico, como es el caso de la gripe (10), en el cual la persona comienza a manifestar síntomas como, tos, fiebre y disnea, sin embargo, en el caso del COVID-19 pueden presentarse complicaciones como neumonía, síndrome de distrés respiratorio agudo entre otros.

La información que llega de Oriente es fragmentada, dispersa e incompleta, el tiempo corre muy rápido, en Occidente el tema casi pasa desapercibido, lo que ayuda a subestimarlo (9), hasta que el virus llega a países como Estados Unidos, España, Italia donde hace sentir su impacto en la salud de los habitantes especialmente en los adultos mayores, colapsando sistemas sanitarios y avizorando un panorama nada alentador de un virus desconocido y sin saber cómo enfrentarlo.

El 30 de enero la Organización Mundial de la salud declara al coronavirus como emergencia global, reportando China 7.711 casos y 170 muertes y la extensión del virus en todas sus provincias (11).

El 27 de febrero se diagnostica el primer caso de COVID-19 en México, a pocas horas se diagnostica un segundo caso positivo en el estado de Sinaloa, ambos pacientes coincidieron en un viaje reciente a Italia (11), $\mathrm{y}$ es que a pesar de estar tomando medidas preventivas con el distanciamiento y el cumplimiento obligatorio de la cuarentena, y la supervisión de los vuelos y la aplicación de medidas de bioseguridad en los pasajeros extranjeros que ingresaban al país, igualmente ocurrió el contagio debido a que el virus puede presentar su primer síntoma hasta los 15 días por lo que posteriormente
ISSN 2773-7705

Periodo. Julio - Diciembre 2021

Vol. 5, Nro. 2, Publicado 2021-12-31

conociendo esto, se procedió a la cancelación de vuelos y al cierre de los aeropuertos no solo México sino que también otros países se sumaron a esta medida.

El 20 de marzo Italia reporta 6000 casos nuevos y 627 muertes en 24 horas. El 26 de marzo Estados Unidos se convierte en el país con el mayor número de casos conocidos en el mundo (12). En marzo del 2020 la Organización Mundial de la Salud lo declaró pandemia mundial, encontrándose la mayoría de países con hospitales colapsados, un aumento elevado de mortalidad, sin saber cómo parar la propagación de este virus e implementando estrategias como el uso de mascarillas, lavado de manos, distanciamiento social y cuarentena para poder contener la situación en la que se encontraban; cada país implemento estas medidas en tiempos diferentes siendo esto evidenciando en las cifras de contagios que cada día aumentaban.

La tendencia evoluciona de tal manera que hacia el 31 de mayo se encuentra una prevalencia global de 5.934.936 casos confirmados, el continente americano alrededor del $66 \%$ de los mismos, con 367.116 defunciones identificadas y con una taza de letalidad global de $6.2 \%$ (13).

La pandemia del COVID-19, ha originado una emergencia sanitaria que ha requerido acción inmediata de los gobiernos, las empresas y las personas (14), encontrándose en una situación crítica en la cual no hay camas disponibles ni suficientes respiradores ni insumos para tratar a todos los pacientes contagiados especialmente aquellos que se encuentran críticos y necesitan soporte vital avanzado.

Según la OMS, actualmente en el mes de agosto existen 20.730.456 casos confirmados a nivel mundial de coronavirus y ha cobrado millones de vida de civiles, así como el personal sanitario que está en la primera línea de lucha, para salvar las vidas de personas contagiadas, y que en el proceso de brindar atención y cuidados han terminado infectados por este virus y han perdido la vida. Ecuador se encuentra en el puesto número 27 a nivel mundial con 98.343 casos de COVID-19 y 6.010 fallecidos según la OMS (13), además de 13.077 casos con alta hospitalaria, 912 hospitalizados estables, 343 hospitalizados con pronóstico reservado y 66.099 pacientes recuperados. El 29 de febrero del 2020 se confirmó el primer caso de coronavirus en el Ecuador proveniente de una mujer de 71 años que llegó de Madrid a Guayaquil (15) así lo manifestó la Ministra de Salud Catalina Andramuño en una rueda de prensa, además de manifestar que se fortalecieron los protocolos de vigilancia en puertos, aeropuertos y fronteras.

Sin embargo, estas medidas no fueron suficientes y a partir del caso cero continuaron confirmándose más casos de COVID-19 hasta el punto de colapsar todo el sistema sanitario de Ecuador, evidenciándose la falta de recursos en las unidades operativas, necesarias para atender a los pacientes que estaban críticos, así como los 
HIGIA DE LA SALUD

que acudían por presentar la sintomatología del coronavirus.

$\mathrm{Y}$ es que, a pesar de las medidas implementadas por el gobierno, gran parte de la población se negaba a acatar dichas normas a pesar de que su incumpliendo acarreaban sanciones, es el caso de Guayaquil la primera ciudad del Ecuador en ser golpeada con uno de los peores brotes de coronavirus.

Según datos del Ministerio de Salud Pública la provincia de Manabí presenta 7.368 casos confirmados con 825 fallecidos, mientras que en la ciudad de Jipijapa presentan 346 casos confirmados de COVID-19 (16). En la actualidad no existe una vacuna contra el SARSCOV2, lo cual hace más rápida y efectiva su propagación y por ende el contagio.

La Organización Panamericana de la Salud en el mes de septiembre del 2020 manifestó que, el continente americano presenta el mayor número de contagios a nivel mundial en el personal de sanitario, registrando 570.000 trabajadores de la salud contagiados por COVID-19 (17). Entre los países de Estados Unidos y México suman $85 \%$ de todas las muertes por coronavirus entre profesionales sanitarios en la región, el Instituto Nacional de Salud de Colombia señala que existe 306 profesionales contagiados hasta el mes de agosto del 2020 (18), mientras que en Ecuador el Ministerio de Salud Pública en el último boletín del mes de mayo del 2020 informo que la cifra de contagios del personal de salud por coronavirus es de 3469 profesionales (19).

El departamento de epidemiología del Distrito de Salud 13D03, según la entrevista realizada, manifestó que existen más de 10 profesionales de la salud los cuales hicieron la respectiva cuarentena y dieron positivo al SARS-COV2, estos recibieron el debido tratamiento y actualmente se encuentran laborando en las respectivas unidades operativas.

\section{Materiales y Métodos.}

El presente trabajo de investigación fue realizado en el Distrito de Salud 13D03, la población utilizada fue el

En el resultado de la pregunta 3 de la encuesta

¿Aplica usted las medidas de bioseguridad en su trabajo?

TABLA 3. APLICACIÓN DE MEDIDAS DE BIOSEGURIDAD

Orden Alternativas Frecuencia

\section{Resultados}

Los resultados de este estudio nos permitieron determinar la eficacia de la aplicación de las normas y su cumplimiento, en la prestación de servicios del personal sanitario para evitar contagios por COVID-19 en el Distrito de Salud 13D03

Es evidente que la aplicación de las medidas de bioseguridad en el ámbito laboral de la población investigada, se cumplen en un $90 \%$, dejando un $10 \%$ de posibilidades de vulnerabilidad que debe de corregirse.

\begin{tabular}{|c|c|c|c|}
\hline $\mathbf{A}$ & $\mathrm{Si}$ & 45 & $90 \%$ \\
\hline B & No & 0 & $0 \%$ \\
\hline C & A veces & 5 & $10 \%$ \\
\hline
\end{tabular}

Fuente: Personal Sanitario del Distrito de Salud 13D03 


\section{HIGÍA DE LA SALUD}

En la siguiente tabla se muestra la aplicación de las medidas de bioseguridad por parte del personal de salud, entre los cuales se evidencia que la gran mayoría de los profesionales aplican las medidas de bioseguridad en su ambiente de trabajo y labores diarias, mientras que una pequeña parte manifestó que solo las aplica a veces, En el resultado de la pregunta 4 de la encuesta ¿Realiza los 5 momentos del lavado de manos? debido a la falta de disponibilidad de insumos y equipos de protección personal en los centros de salud, mientras que otros manifestaron que esto se debe a que existe limitación por parte del distrito en la utilización de los EPP completos de manera conjunta y continua.

\section{TABLA 4. APLICACIÓN DE LOS 5 MOMENTOS DEL LAVADO DE MANOS}

$\begin{array}{llll}\text { Orden Alternativas } & \text { Frecuencia } & \text { Porcentaje }\end{array}$
A
$\mathrm{Si}$
46
$92 \%$
B
No
0
$0 \%$
C
A veces
4
$8 \%$
Total

Fuente: Personal Sanitario del Distrito de Salud 13D03

Las manos son el principal instrumento de trabajo del personal sanitario, con ellas se brinda la atención a los pacientes, se realizan procedimientos y un sin número de acciones que contribuyen a su recuperación, sin embargo, también pueden convertirse en un medio de peligro porque constituyen la principal vía de transmisión de microorganismos causantes de infecciones, como podemos observar en la tabla la mayoría de profesionales aplican los 5 momentos de lavados de manos, 2 antes y 3 después que protegen tanto al paciente como al profesional, mientras que una minoría los realiza solo a veces por lo cual se debe poner énfasis en la importancia de un correcto lavado de manos como medida preventiva principal para combatir la trasmisión de microorganismos especialmente por la pandemia actual del coronavirus.

En el resultado de la pregunta 7 de la encuesta

¿Existe disponibilidad de insumos y equipo de protección necesario para realizar los respectivos procedimientos en su área de trabajo?

TABLA 7. DISPONIBILIDAD DE INSUMOS Y EPP

Orden

A

B

C

A veces

Total Alternativas

$\mathrm{Si}$

No

50

Fuente: Personal de Sanitario del Distrito de Salud 13D03

El equipo de protección personal proporciona un medio de barrera que otorga protección contra agentes patógenos, es de suma importancia su utilización y disponibilidad en las unidades de salud para la realización de las diversas actividades asistenciales realizadas por los profesionales sanitarios. Como se En el resultado de la pregunta 8 de la encuesta ¿Además de la mascarilla qué equipos de bioseguridad utiliza a diario, para protección contra el COVID-19? TABLA 8. EQUIPOS DE BIOSEGURIDAD USADOS DIARIAMENTE
Frecuencia Porcentaje

$46 \quad 92 \%$

$0 \%$

$48 \%$

$100 \%$ puede observar gran parte de los encuestados manifestaron que en sus respectivas unidades operativas si cuentan con el EPP necesario, mientras que una parte manifestó que no existe la suficiente disponibilidad y acceso a estos insumos. 


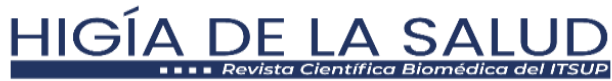

Orden

Alternativas

A

Gorro

B

Protectores Faciales

C

D

$\mathbf{E}$

F

Guantes

Gafas

Batas desechables

Trajes de protección

Total
Frecuencia

$\begin{array}{cc}14 & 28 \% \\ 0 & 0 \% \\ 32 & 64 \% \\ 4 & 8 \% \\ 0 & 0 \% \\ 0 & 0 \% \\ 50 & 100 \%\end{array}$

Fuente: Personal Sanitario del Distrito de Salud 13D03

La mascarilla es el principal elemento de protección personal de uso obligatorio para toda la población, así como en el personal de salud, a parte de este dispositivo existen otros equipos indispensables y de uso específico para la protección del personal, entre los cuales, los guantes son la prenda de protección más utilizada, seguida de los gorros y las gafas de protección, en la En el resultado de la pregunta 9 de la encuesta

¿Considera usted que la aplicación de los lineamientos de prevención y control para casos SARS COV-2 implementados por el MSP son eficaces?

TABLA 9. EFICACIA DE LOS LINEAMIENTOS PARA CASOS SARS-COV2

Orden

Alternativas

$\mathrm{Si}$

No

Total

Fuente: Personal Sanitario del Distrito de Salud 13D03

Como se puede observar en la tabla 10, gran porcentaje de los encuestados manifestaron que son eficaces la aplicación de los lineamientos, mientras que un menor número expresa que no lo son. En la entrevista realizada a la Lcda. Loor del departamento de epidemiología, manifiesta que el Distrito 13D03 ha cumplido a cabalidad cada uno de los protocolos según las disposiciones del MSP, mientas que la Lcda. Velasteguí En el resultado de la pregunta 11 de la encuesta ¿Se ha contagiado de COVID-19?

TABLA 11. CONTAGIO POR COVID-19 Orden Alternativas
Frecuencia

40

10

50

entrevista realizada la Lcda. Velastegui manifestó que el uso del equipo de protección personal completo como batas desechables, gafas, gorro, mascarillas KN95 son utilizadas exclusivamente para atender casos sospechoso o confirmados de coronavirus, más no en la atención diaria de los pacientes.
$\mathrm{Si}$
No
A
15
$30 \%$
B

$35 \quad 70 \%$

expuso que sin la utilización del equipo de protección personal descartable y de uso diario, no garantiza la seguridad de los profesionales y si el MSP estuviera defendiendo a capa y a espada al personal de salud dotará correctamente de estos insumos para evitar contagios masivos no solamente cuando se encuentren casos positivos o sospechosos de que se atienden pacientes con COVID-19.
Frecuencia

\section{Porcentaje}


Fuente: Profesionales Sanitarios del Distrito de Salud 13D03

El Ministerio de Salud Pública en el último boletín del mes de mayo del 2020 informó que la cifra de contagios de personal de salud por coronavirus es de 3469 En el resultado de la pregunta 12 de la encuesta ¿En qué escenario se infectó por coronavirus? TABLA 12. ESCENARIO DE CONTAGIO POR CORONAVIRUS

Orden Alternativas profesionales de salud (19), en las encuestas realizadas a los 50 profesionales que se tomaron de muestras, 15 se han contagiado por coronavirus.
Consulta medica
Preparación de pacientes
C
D
$\mathbf{E}$
Otros
Total
A
Realización de procedimientos
0
6
$40 \%$
B
Actividades extramurales
6
$40 \%$
0
$0 \%$
$100 \%$
$20 \%$
$0 \%$

Fuente: Profesionales Sanitarios del Distrito de Salud 13D03

De 15 profesionales que dieron positivo al SARSCOV2, el escenario con mayor prevalencia donde contrajeron el virus fue en la consulta médica y en la realización de las actividades extramurales, además un

En el resultado de la pregunta 13 de la encuesta

¿Cuál considera usted que fue la causa probable de contagio por coronavirus?

TABLA 13. CAUSA PROBABLE DE CONTAGIO POR CORONAVIRUS

\section{Orden}

A

B

C

D

$\mathrm{E}$

Total
Alternativas

Uso incorrecto de mascarilla

Incumplimiento de normas de distanciamiento

Realización de procedimientos invasivos sin precaución

Falta de disponibilidad de equipos de protección

Actividades extramurales sin las debidas medidas de
Frecuencia

4

0

0

\section{Porcentaje}

pequeño grupo de profesionales manifestaron que se contagiaron al momento de realizar la preparación de pacientes en las unidades operativas. personal en la unidad operativa bioseguridad

\section{Porcentaje \\ $27 \%$ \\ $0 \%$}

$6 \quad 40 \%$

$5 \quad 33 \%$

15
$100 \%$

Fuente: Profesionales Sanitarios del Distrito de Salud 13D03

En la siguiente tabla se muestran las posibles causas por las que se contagiaron el personal sanitario, entre las cuales encontramos la falta de disponibilidad de equipos de protección personal en la unidad operativa que se evidenciaron al comienzo de la pandemia, la realización de actividades extramurales sin las medidas de 
HIGIA DE LA SALUD

bioseguridad y el uso incorrecto de la mascarilla, con respecto a este tema la licenciada Velasteguí expreso que a inicios de la pandemia ninguna unidad operativa estaba preparada para enfrentar tal acontecimiento de los hechos que actualmente se han suscitado, por lo que se tenía que laborar con los insumos disponibles y manejarlos de la manera racional, además que no se tenía conocimiento de este nuevo virus por lo que no se pudo tomar las medidas necesarias a tiempo para prevenir contagios masivos tanto en la población como Discusión

Otras investigaciones realizadas recientemente, afirman la importancia del manejo de la infección por SARSCOV-2/COVID-19 en establecimientos de atención de la salud (20); haciendo énfasis en la importancia del uso de las barreras de protección y la aplicación de las normas de bioseguridad en las diferentes áreas hospitalarias.

De acuerdo a los estudios realizados en España, se establece, como debemos de comportarnos ante situaciones como la pandemia actual, sin disponer de los recursos adecuados (21).

Podemos citar que de acuerdo a la investigación realizada, es necesario fomentar la correcta aplicación de las normas de bioseguridad en el personal del Distrito de Salud 13D03, enfatizando en el correcto de lavado de manos y la aplicación de los 5 momentos como medida preventiva para evitar la trasmisión de microorganismos patógenos y precautelar la salud tanto del paciente como de los profesionales logrando que todo el personal sanitario cumpla correctamente con las medidas de bioseguridad, y que se les proporcione los insumos y equipos de bioseguridad necesarios sin limitaciones para asegurar la protección adecuada y minimizar los contagios por coronavirus en el personal de salud. Además, que tomen en consideración las causas por las que los profesionales sanitarios se contagiaron de COVID-19 e implementen medidas que minimicen el riesgo de contagio sin perjudicar el rendimiento y desempeño laboral de los mismos.

La presente investigación es relevante ya que aporta a nuestra sociedad en busca de soluciones al problema planteado buscando mejorar la atención integral de los pacientes y la calidad de vida de la sociedad. En la actualidad si se han realizado más estudios sobre el tema investigado, ya que es una problemática no solo a nivel local, sino a nivel mundial.

Conclusiones
ISSN 2773-7705

Periodo. Julio - Diciembre 2021

Vol. 5, Nro. 2, Publicado 2021-12-31

el personal de salud, actualmente se tiene mayor conocimiento e información sobre el SARS-COV2 igualmente se ha abastecido a las unidades operativas con los debidos insumos necesarios. La licenciada Loor manifestó que actualmente todo el personal sanitario que se contagió en la pandemia realizó la debida cuarentena, recibieron el debido manejo y actualmente se encuentran laborando en sus respectivas unidades operativas.

Mediante la aplicación de las técnicas de recolección de datos se obtuvo los siguientes resultados en donde se pudo identificar lo siguiente:

a) Se logró conocer que el personal sanitario que labora en el Distrito de Salud 13D03 tiene el conocimiento necesario sobre las normas de bioseguridad en su práctica profesional, la mayoría de los participantes de esta investigación cumplen con las debidas normas de bioseguridad precautelando su seguridad y la del paciente, siguiendo las normas y protocolos implementados por el Ministerio de Salud Pública evitando así contagios por COVID-19, sin embargo una minoría no las cumple correctamente debido a las limitaciones en la accesibilidad de equipos de protección personal.

b) La aplicación de las normas de bioseguridad son sin duda un parte esencial para la lucha contra el coronavirus, desde el lavado de manos y la correcta aplicación de los 5 momentos, respeto de las normas de distanciamiento, utilización del equipo de protección personal como por ejemplo mascarillas, guantes, gorro que son los equipos más utilizados en la labor diaria del personal sanitario, mientras que para la atención de un paciente sospechoso por coronavirus la utilización del EPP completo es esencial para precautelar la salud del mismo y evitar contagios masivos por coronavirus.

c) El SARS-COV2 es un virus altamente virulento y los profesionales de la salud son uno de los grupos con más riesgos de contagiarse con coronavirus debido a que se encuentran en primera línea de lucha, atendiendo pacientes que acuden a las unidades asistenciales por diversas patologías, entre las causas por las que los profesionales contrajeron el virus del COVID-19 se encuentra la falta de disponibilidad de equipos de protección personal en las unidades operativas, el uso incorrecto de la mascarilla, así como la realización de actividades extramurales sin las debidas medidas de bioseguridad, es aquí donde se evidencia la importancia de la disponibilidad de insumos para el personal de salud así mismo como la aplicación correcta de las normas de bioseguridad. 


\section{Bibliografía}

1. Aguilar RE, González Sanchez J, Morchon R, Martines Merino V. ¿Seguridad biológica o bioseguridad laboral? Scielo. 2015 Julio 07;(472-277).

2. Romero Ramírez HA. Relación entre el cumplimiento de normas de bioseguridad e incidencia de infección de la herida quirúrgica. Tesis Doctoral. Lima: Universidad Nacional Mayor de San Marcos, Departamento de Cirugia Hospital del Seguro Social Babahoyo; 2017.

3. Padilla Languré, García Puga, Salazar Ruibal, Quintana Zavala O. Normas de biosegurdad del personal de enfermería en una Institución Hospitalaria. Revista de Ciencias Biológicas y de la Salud. 2016 Enero 25; XVIII.

4. Ortiz, Antonietti , Capriati A, Ramos, Romero, Mariani J. Preocupaciones y demandas frente al covid-19. Encuesta al personal de Salud. Medicina Buenos Aires. 2020; VI(8).

5. Verelst, Kuylen, Beutels. Indications for healthcare surge capacity in European countries facing an exponential increase in coronavirus disease (COVID-19) cases, March 2020. Eurosurveillance. 2020 Abril.

6. Panchi Sinchiguano LA. Estrategias de intervención para la aplicación adecuada de las medidas de bioseguridad por el personal de enfermería durante la estancia hospitalaria del paciente en el Hospital Básico de la base area Cotopaxi. Ambato: Universidad Regional Autonoma de los Andes, Departamento de Ciencias Medicas; 2019.

7. Ministerio de Salud Pública. salud.gob.ec. [Online].; 2020 [cited 2020 Agosto 7. Available from: https://www.salud.gob.ec/documentos-informativos-covid-1ecuador/.

8. Haiyang Y, Xu Han , Guanlan Zhao. Aspectos Importantes de prevención del personal sanitario en los Hospitales durante la epidemia del nuevo coronavirus 2019. Rev.Esp.Salud Publica. 2020 Julio 2; 94: p. 7.

9. Rodríguez Gutiérrez CH. Tributos en tiempo de Pandemia COVID-19, Nuevas realidades e imperiosas exigencias, grandes retos futuros para la sociedades y los estados. Academia Colombiana de Jurisprudencia. 2020 Junio;(371).

10. Rodríguez Monera E. Bioseguridad y Covid-19. Informe de recomendaciones ROE. España: Registro de Osteopatas de España; 2020.

11. Villagrán Olivas KA, Torrontegui Zazueta LA, Entzana Galindo. Características clínico-epidemiológicas de pacientes de COVID-19. Revista Medica UAS. 2020 Junio; 10.

12. Hoffman C, Kamps S. Covid Reference. Fourth Edition ed. Camp, editor.:

Steinhauser Verlag; 2020.

13. Salud OMdl. Who.int. [Online].; 2020 [cited 2020 Agosto 7. Available from: https://covid19.who.int/table.

14. Gomez Ortega M. Bioseguridad en el personal de salud en tiempos de pandemia. Mexico: Universidad de Sonora, Departamento de Enfermeria Y Obstetricia ; 2020. Report No.: SANUS/ISSN.

15. Ministerio de Salud Pública. salud.gob.ec. [Online].; 2020 [cited 2020 Agosto 15. Available from: https://www.salud.gob.ec/ministra-de-salud-informa-sobresituacion-actual-del-coronavirus-en-ecuador/.

16. Ministerio de Salud Publica. Salud.gob.ec. [Online].; 2020 [cited 2020 Agosto 15. Available from: https://www.salud.gob.ec/el-ministerio-de-salud-publicadel-ecuador-msp-informa-situacion-coronavirus/.

17. Organización Panamericana de la Salud. [Documento].; 2020 [cited 2020 Septiembre 3. Available from: https://www.paho.org/es/documentos/atencion-altrabajador-salud-expuesto-al-nuevo-coronavirus-covid-19establecimientos. 


\section{HIGÍA DE LA SALUD}

Periodo. Julio - Diciembre 2021

18. Instituto Nacional de salud Colombia. Datos de Personal de salud contagiado por Covid 19 [Documento pdf]. Bogota

; $2020 \quad$ [cited $2020 \quad$ Septiembre

https://www.ins.gov.co/COVID19PERSONALDESALUDCOLOM-

BIA/Datos-COVID-19-personal-salud-240420.jpg.

19. Ministerio de Salud Pública . [Boletin].; 2020 [cited 2020 Septiembre 3. Available from: https://www.edicionmedica.ec/secciones/profesionales/3-469casos-positivos-aumenta-el-numero-de-profesionalesde-salud-contagiados-decovid19-95769.

20. Saavedra Trujillo C, Consenso colombiano de atención, diagnóstico y manejo de la infección por SARS-COV2/COVID-19 en establecimientos de atención de la salud. Recomendaciones basadas en consenso de expertos e informadas en la evidencia, Revista Scielo, Bogotá-Colombia, 2020.

21. Serrano-Cumplido A, Eguía Ortega A, COVID-19. La historia se repite y seguimos tropezando con la misma piedra, Science Direct, Volumen 46, Suplemento 1 , agosto de 2020 , páginas 48-54 